\title{
Study of preventive health measures for wildlife in captivity: a review of management approaches
}

\begin{abstract}
The foundation of a medical program for zoo animals is preventive medicine. Preventive medical programs should be adaptive and include attention to individual specimens as well as the herd, troop, or flock. Components of the program include quarantine of new arrivals, periodic fecal examinations and treatments for parasites, booster vaccinations, health screening procedures, nutrition evaluation, necropsy examination of deceased specimens, and a comprehensive pest control program. Animals should be evaluated to ensure their health complies with local, state, and federal health requirements before shipment to other zoos or before release in managed reintroduction programs. Preshipment evaluations can also be used as an opportunity to assess the overall health status of the group in which the animal has been living. The present review study is illustrating the importance of the preventive measure used for wildlife into the captivity and the management and tactics to achieve this.
\end{abstract}

Volume 2 Issue 3 - 2017

\author{
Bais B, Tak L, Mahla S \\ Department of Livestock Products Technology, Rajasthan \\ University of Veterinary and Animal Sciences, India
}

\begin{abstract}
Correspondence: Bais B, Department of Livestock Products Technology, College of Veterinary and Animal Sciences, Rajasthan University of Veterinary and Animal Sciences, Bikaner, Rajasthan33400I, India, Email basantbais@gmail.com
\end{abstract}

Received: June 20, 2017 | Published: August 07, 2017

Keywords: wildlife, captivity, animals, management

\section{Introduction}

A captive animal is the wild animal, which is held under confinement and is dependent on humans for provision of all its needs, whereas Captive wildlife facility is includes a sanctuary, an orphanage or a rescue centre that provides shelter and care to animals that have been abused, injured or sick, abandoned or orphaned, illegally held or are otherwise in need. Such a facility may be private, public or community owned. In the course of wildlife conservation and management, some individual animals get orphaned, sick, injured or otherwise incapacitated necessitating interventions to assure them of a life. In many cases during the course of treatment and care, these animals get habituated making it extremely difficult if not impossible to rehabilitate them back to the wild. Consequently, captive animal facilities become the homes for these individuals for purposes of nurturing them. The welfare of each individual animal in these facilities is of primary concern. Management of animals in captivity poses serious challenges that range from animal welfare considerations, space requirements, human skills, veterinary care and visitor satisfaction to financial requirement. ${ }^{1}$

Management of captive wildlife is broad and specialised. Whilst there are many examples of good practice, some captive wild animals suffer because of poor welfare standards. Compounding this is that some animal facilities are unregulated and uncontrolled. Some of these are poorly designed, managed and maintained and are unable to provide the barest essentials necessary for the health and psychological well-being of the animals under their care. The most common shortcomings in captive facilities are: undersized and 'barren' cages and enclosures, unsuitable floor surfaces that can be injurious to the animals and also difficult to clean to maintain good hygiene, poor quality feed and nutrition, inadequate veterinary/health care and insufficient expertise in appropriate animal care and in some instances ignorance of legal provisions. ${ }^{2}$ With defined minimum standards for management of wildlife in captivity in place, many of the challenges that face captive facilities will be addressed. To know all these factors and for better management purpose, some of the good practices are:

\section{Quarantine}

Animals entering a collection must undergo quarantine. Quarantine facilities should be designed to allow handling of animals and proper cleaning and sanitizing of enclosures. Shipping crates should be cleaned and disinfected before they leave the quarantine area, and the crates' contents disposed of appropriately. Quarantine facilities require barriers against ingress of potential vectors and vermin. Separate keepers who are skilled at recognizing signs of stress and disease and who will carefully monitor feed intake and fecal characteristics should care for quarantined animals.

Quarantine entry should be strictly controlled. Only essential personnel should be allowed into the quarantine facility. Individuals leaving the quarantine facility should not return to other animal areas without showering and changing clothing. The duration of quarantine should be appropriate to ensure that infectious diseases are not introduced into the permanent collection when the quarantined animals are released to exhibits. Quarantine facilities should follow the "all-in/all-out" principle, i.e., if additional animals are added to an ongoing quarantine, the quarantine period should be restarted.

During quarantine, animals should receive appropriate vaccinations and diagnostic testing (eg, tuberculosis, heartworm). They should be examined and treated for ecto- and endoparasites and screened for enteric bacterial pathogens. Before release, animals should receive physical and laboratory examinations, which may include radiographs, serology, hematology, and clinical chemistries. Serum should be frozen for future reference and possible epidemiologic studies. All procedures and results should be recorded in each individual animal's medical record, which is an essential component of the medical program. Each animal should also be identified by some permanent method (eg, tattoo, tag, band, eartag, transponder) to ensure future identification.

When new animals are introduced to enclosures, caution and forethought are necessary to prevent self-induced trauma. Visual barriers, eg, suspending canvasses from fences or enclosure walls or obscuring glass with soap to provide a visual cue, are standard 
management steps to protect newly introduced specimens from accidents during acclimation to a new exhibit. ${ }^{3}$

\section{Parasite control}

Like domestic animals, zoo animals are vulnerable to a wide variety of ecto and endoparasites, and similar drugs are used for treatment. Care must be exercised in the choice of medications due to species-specific sensitivities to some drugs. Young animals and those stressed by shipment, disease, or injury are the most likely to be adversely affected by parasites. At these times, commensal parasites (especially protozoa) can cause disease. Acute diarrhea can result from massive infections of Coccidia, Trichomonas, Giardia, or Balantidium spp. Amebiasis, which is fairly common in primates and reptiles, can be fatal in a compromised animal. ${ }^{4}$ Intestinal parasites may be a major, continuous problem in species kept in naturalistic exhibits or on dirt substrate or pasture, especially in young, newly introduced, or stressed individuals. Of most concern are parasites with direct life cycles. Incorporating anthelmintics directly into the feed is helpful. As in domestic species, anthelmintic resistance may develop and necessitate rotating medication. Parasites with indirect life cycles are less frequently a problem if the exhibit area is free of intermediate host.

\section{Vaccination}

Vaccination programs for carnivores, nonhuman primates, equids, artiodactylids, and birds should be developed. Vaccination of zoo carnivores is essential because of their susceptibility to various diseases such as feline panleukopenia, feline rhinotracheitis, feline calicivirus, rabies, canine distemper, and canine parvovirus. Previously, only killed virus vaccines were recommended, but recent studies have shown that some modified live vaccines are safe for use in selected species. Further studies are required because some modified live vaccines (especially canine distemper) produce fatal disease in certain species. A canarypox-vectored recombinant canine distemper vaccine has proven safe for use in those species susceptible to modified live virus vaccine-induced disease. Appropriateness of rabies vaccination depends on the circumstances of each collection. If indicated in rabies-endemic areas for the protection of individual animals, only a killed rabies vaccine should be used. The decision to vaccinate zoo animals for less common diseases for which a vaccine is available should be made on an individual basis. Newer recombinant and subunit vaccines are being developed for a variety of infectious diseases for domestic animals and humans. These vaccines should be used with caution until safety and efficacy studies have been completed for zoological species. ${ }^{5}$

\section{Necropsy}

All dead animals should be necropsied. This should include gross and histopathologic evaluation of tissue and viral, bacterial, or fungal cultures when appropriate. Tissues should also be saved for potential future examinations. A thorough pathology examination allows evaluation of medical, management, and nutritional programs. It is also valuable in identifying problems requiring immediate action to safeguard the health of the collection. Variations in anatomy should be recorded because such observations may aid in future diagnostic procedures or therapy in the species.

\section{Pest control}

A successful control program is continuous and requires a concerted effort by zoo staff to minimize harborage and food for pests, in addition to the use of mechanical and chemical control methods. Choice of agent, method of use, and storage may minimize zoo animals' access to pesticides and the risk of secondary poisoning. Common zoo pests may serve as important disease vectors. For example, cockroaches are intermediate hosts for GI parasites of primates and birds; rodents can harbour and spread Listeria, Salmonella, and Leptospiras $p p$ and Francisella tularensis. ${ }^{6}$ Wild and feral carnivores such as foxes, raccoons, and domestic dogs and cats can devastate animal collections through predatory attacks and may be important vectors for viral diseases such as rabies, parvovirus, and canine distemper. Raccoons may also transmit Baylisascaris parasites, which can cause larval migration resulting in fatal neuropathy in some species. Pigeons, geese, ducks, and starlings are potential reservoirs for avian diseases; they consume or contaminate animal food and deposit droppings everywhere. Arthropod vectors can transmit pathogens such as West Nile virus.

\section{Dental health}

Carnivores and primates often accumulate tartar on their teeth just like humans do. This requires that the animal's teeth be cleaned on a regular basis (the tartar removed and the teeth cleaned and polished). Occasionally, more serious problems require more complex procedures such as root canals. ${ }^{7}$

\section{Neonate examination}

Newborn animals at Zoo should be examined to check their health, to give those vitamins, their first vaccinations and some kind of individual identification such as an ear tag or microchip. The signs of hungry status are frequent making of sound, when hungry; the calf often attempts to suckle the tip of the finger kept near the mouth. When stomach is full, the neonates tend to go for sleep with snoring sounds that are well audible.

Mode of feeding: Large sized enema or a big-sized bottle like container with tube may be used and approximately ten per cent of the body weight may be taken as a rough estimate of feed requirement. Regular nipples attached to bottles of adequate volume or buckets have worked well for the successful feeding of orphaned elephant calf. $^{8}$

\section{Husbandry and nutrition}

Nutrition standards speak to the basic need to nourish the animals in a manner that ensures their continued physical health. Access at all times to clean water, for example, is essential. Equally important, however, and widely acknowledged, is the requirement that a diet be tailored to the species and include a broad variety in the types of food provided to enhance the captive animal's quality of life. ${ }^{9}$ A zoo veterinarian has to evaluate and approve husbandry and assist in developing nutrition programs for each species within a collection. Each animal must receive a timely supply of wholesome and unadulterated food insufficient quantity, according to the requirement of each individual. Potable water must be available around the clock in each enclosure.

\section{Personnel health program}

A well-defined personnel health policy is also an important part of a preventive health program. Several infectious diseases of humans, such as tuberculosis, measles, and amoebic dysentery, can be acquired by captive wild mammals, especially primates. Pre-employment screening and proper training, plus periodic tuberculin testing and 
health monitoring during employment, will minimize the potential for disease transmission from caretakers to animals.

\section{Conclusion}

In India, enclosures must be designed to meet the full biological requirements of the animals they contain. In particular, the following are required: space for free movement and exercise; no undue domination by individuals within herds or groups; no unnatural provocation for public benefit; no stress caused by visibility of others in adjoining enclosures; to endeavour to simulate conditions of natural habitat; trees for shade and shelters to be constructed to merge with the environment. They must have resting and exercising facilities tailored to meet the biological needs of the species. They must also have proper ventilation and lighting. Animals must be kept in viable social groups. Facilities may not acquire a single animal of any species with an exception related to specific breeding issues. Many of the jurisdictions we examined have implemented thorough requirements with respect to the care that must be given to captive wildlife and the safety of the people who come into contact with them. Most significantly, it is clear from an enforcement perspective that emphasis on a stringent, preventative application process is desirable.

\section{Acknowledgements}

None.

\section{Conflict of interest}

The author declares no conflict of interest.

\section{References}

1. Audigé L, Doherr MG, Hauser R, et al. Stochastic Modelling as a Tool for Planning Animal-health Surveys and Interpreting Screening-test Results. Prev Vet Med. 2001;49(1-2):1-17.

2. Woodroffe R. Managing Disease Threats to Wild Mammals. Animal Conservation. 1999;2(3):185-193.

3. Martin SW, Meek AH, Willeberg P. Veterinary Epidemiology: Principles and Methods. USA: Iowa State University Press; 1987. 343 p.

4. Scott ME. The Impact of Infection and Disease on Animal Populations: Implications for Conservation Biology. Conservation Biology. 1988;2(1):40-56

5. Eric Miller R, Murray E Fowler. Fowlers Zoo and Wild Animal Medicine Current Therapy. 1st ed. 2014. 8:792 p.

6. Leighton FA. Health Risk Assessment of the Translocation of Wild Animals. Rev sci tech Off int Epiz. 2002;21(1):187-195.

7. Ekboir JM. The Role of the Public Sector in the Development and Implementation of Animal Health Policies. Preventive Veterinary Medicine. 1999;40(2):101-115.

8. Devra G Kleiman, Katerina V Thompson, Charlotte Kirk Baer. Wild Mammals in Captivity: Principles and Techniques for Zoo Management. 2nd ed. USA: The University of Chicago Press; 2010. 592 p.

9. Ahl AS, Acree JA, Gipson PS, et al. Standardization of Nomenclature for Animal Health Risk Analysis. Rev sci tech Off int Epiz. 1993;12(4):10451053. 